\title{
Tail behaviour of a general family of control charts
}

\section{Wolfgang Schmid, Yarema Okhrin}

Received: May 29, 2002; Accepted: January 21, 2003

\begin{abstract}
Summary: In this paper we consider a general control scheme. The control statistic $Z_{t}$ is equal to an arbitrary weighted sum of the past observations $X_{t}, \ldots, X_{1}$. This approach covers most of the applied control schemes like for instance moving average, EWMA and ARMA $(1,1)$ charts. The process $\left\{X_{t}\right\}$ is assumed to be a stationary Gaussian process. The aim of the work is to analyze the behaviour of the tail probability of the run length $N=\inf \left\{t \in \mathbb{N}: Z_{t}-E\left(Z_{t}\right)>c \sqrt{\operatorname{Var}\left(Z_{t}\right)}\right\}$ with respect to the autocorrelation of $\left\{X_{t}\right\}$. It is shown under which conditions on the weights and on the autocorrelations of $\left\{X_{t}\right\}$ the correlation between $Z_{t}$ and $Z_{t-i}$ is a nondecreasing function in the autocorrelations of the observed process. Using this result it can be proved that the probability of a false alarm is a nondecreasing function of the autocorrelations of $\left\{X_{t}\right\}$, too. The weight conditions are verified for several well-known charts.
\end{abstract}

\section{Introduction}

Various control charts have been developed as tools of statistical process control (SPC). Initially they rested on the assumption of independency of the underlying process $\left\{X_{t}\right\}$. In the last years the methods of SPC are becoming of greater importance in many fields like e.g. economics, medicine, etc. For that reason it was necessary to extend the control chart technique to time series data. Nowadays, two possible solutions are available for autocorrelated processes. The first, residual charts, suggests to transform the process to achieve independency and afterwards classical charts are applied (e.g. $[1,2,12])$. Here we deal with modified control charts. For mean charts the control statistic $Z_{t}$ can be regarded as a predictor of $X_{t+1}$ given $X_{t}, \ldots, X_{1}$. This quantity is compared with the target value of the mean. If the deviation is larger than a multiple $c$ of the standard deviation of the process it is concluded to be out-of-control. This means that there is a change in the mean behaviour of the observed process. In practice, simple predictors are applied. The Shewhart chart is based on $Z_{t}=X_{t}$ while the exponentially weighted moving average (EWMA) chart makes use of $Z_{t}=(1-\lambda) Z_{t-1}+\lambda X_{t}$ (see [6,7]). The $\operatorname{ARMA}(1,1)$ statistic $Z_{t}=\alpha_{1} Z_{t-1}+\beta_{0} X_{t}+\beta_{1} X_{t-1}$ is considered recently in [3].

A measure for the performance of a control chart is the run length. The run length is equal to the first value at which it is concluded that the process is out-of-control. The

AMS 1991 Subject Classifications: Primary 62N10; secondary 62L10; third 60G10

Key words and phrases: control charts; multivariate normal distribution; run length; statistical process control; tail probability; time series 
most often used characteristic is the first moment of the run length - the average run length (ARL). A 'good' control chart is characterized by a high in-control ARL and a low out-of-control ARL. The impact of the strength of autocorrelation on the behaviour of the ARL has to be analyzed. In [9] it is proved that the in-control ARL of the one-sided EWMA chart for a series with positive autocorrelation is higher than for independent observations. The practical consequence is, that if the process is falsely assumed to be independent, then the actual ARL is underestimated and the process is uselessly interrupted. In [10] this result is extended and it is shown that for a stationary Gaussian process $\left\{X_{t}\right\}$ the probability of no signal until a fixed time is a nondecreasing function in the autocorrelations. What happens if the Shewhart chart is applied to a nonlinear time series is investigated in [5]. They proved that for GARCH processes a similar property only holds under an additional restriction on the control limits. Such a condition was not necessary for Gaussian processes.

Many control charts have one important feature in common. Their control statistic can be interpreted as a linear one step ahead predictor of the underlying process. Consequently $Z_{t}$ is a weighted sum of the previous observations, i.e. $Z_{t}=\sum_{v=1}^{t} w_{t v} X_{v}$, where $\left\{X_{t}\right\}$ is assumed to be a stationary Gaussian process (see [4]). For instance the weights of the EWMA chart are given by $w_{t v}=\lambda(1-\lambda)^{t-v}$. Due to the starting problem $\left\{Z_{t}\right\}$ is usually not a stationary process. The aim of this paper is to extend the results of [9] and [10] to general control charts of the above described type. The paper is structured as follows. Chapter 2 presents the main results. Theorem 2.1 establishes general conditions for $\operatorname{Corr}\left(Z_{t}, Z_{t-i}\right)$ to be a nondecreasing function in the autocorrelations of the initial process $X_{t}$. Theorem 2.3 provides corresponding restrictions on the autocorrelation function of $\left\{X_{t}\right\}$ and on the weights. This result immediately implies that for a large family of control charts the tail probability of the run length is a nondecreasing function in the autocorrelations of $\left\{X_{t}\right\}$. This is an extension of the result in [10] to an arbitrary weighting scheme. The restrictions on the weights are sometimes hard to check. In applications, however, frequently we have that $w_{t v}=K(t-v)$ with a kernel $K$. For this case the verification of the weight conditions is much simpler (see Proposition 2.5). In Chapter 3 several examples are considered. It is shown how the above results can be applied to the moving average chart with variable weights, the EWMA chart, and the ARMA $(1,1)$ chart. The appendix contains the proofs of all theorems and all propositions.

\section{Main results}

Let $\Delta_{k}$ and $\Gamma_{k}$ be $k$-dimensional covariance matrices. Let $\tau \in[0,1], C_{k}(\tau)=(1-$ $\tau) \Delta_{k}+\tau \Gamma_{k}$. Furthermore let $\mathbf{X}_{k}=\left(X_{1}, \ldots, X_{k}\right)^{\prime}$ be normally distributed with mean 0 and covariance matrix $C_{k}(\tau)$, briefly $\mathbf{X}_{k} \sim \mathcal{N}\left(0, C_{k}(\tau)\right)$. Moreover let $Z_{t}=\mathbf{w}_{t}^{\prime} \mathbf{X}_{t}$ for $t=1, \ldots, k$ with a weight vector $\mathbf{w}_{t}=\left(w_{t 1}, \ldots, w_{t t}\right)^{\prime}$ whose components are all nonnegative. Our aim is to characterize the distribution of $\mathbf{Z}_{k}=\left(Z_{1}, \ldots, Z_{k}\right)^{\prime}$ as a function of $\tau$. Such results are of importance in statistical process control. Because $\mathbf{Z}_{k}$ is again normally distributed Slepian's inequality (cf. [11]) implies that it is sufficient to study the behaviour of its correlation matrix. The main results of our paper are stated as Theorem 2.1 and Theorem 2.3. 
We use the notation $\operatorname{Var}_{\tau}, \operatorname{Cov}_{\tau}, \operatorname{Corr}_{\tau}, P_{\tau}$ to refer to the situation that $\mathbf{X}_{k} \sim$ $\mathcal{N}\left(0, C_{k}(\tau)\right)$. Thus $\operatorname{Cov}_{\tau}\left(Z_{t}, Z_{t-i}\right)=\mathbf{w}_{t}^{\prime} C_{t}(\tau) \tilde{\mathbf{w}}_{t}$ with $\tilde{\mathbf{w}}_{t}^{\prime}=\left(\mathbf{w}_{t-i}^{\prime}, 0, \ldots, 0\right)$. Note that $\tilde{\mathbf{w}}_{t}$ depends on $i$, but for reasons of simplicity this subscript is suppressed. First, we will deal with the question under which conditions on the autocorrelations and on the weights it can be shown that the correlation of $Z_{t}$ and $Z_{t-i}$ is a nondecreasing function in $\tau$.

In the following the symbol $A \geq B$ used for quadratic matrices $A=\left(a_{i j}\right)$ and $B=\left(b_{i j}\right)$ means that $a_{i j} \geq b_{i j}$ for all $i, j$.

Theorem 2.1 Let $\Gamma_{t} \geq \Delta_{t}$. Let $i \in\{0, \ldots, t-1\}$ be fixed, $\operatorname{Cov}_{0}\left(Z_{t}, Z_{t-i}\right)>0$, and

$$
\mathbf{w}_{t}^{\prime}\left(\Gamma_{t}-\Delta_{t}\right) \tilde{\mathbf{w}}_{t}>0 \text { if } \Gamma_{t} \neq \Delta_{t} .
$$

Then $\operatorname{Corr}_{\tau}\left(Z_{t}, Z_{t-i}\right)$ is a nondecreasing function in $\tau$ if and only if

$$
2 \frac{\operatorname{Corr}_{1}\left(Z_{t}, Z_{t-i}\right)}{\operatorname{Corr}_{0}\left(Z_{t}, Z_{t-i}\right)} \geq \sqrt{\frac{\operatorname{Var}_{1} Z_{t} / \operatorname{Var}_{0} Z_{t}}{\operatorname{Var}_{1} Z_{t-i} / \operatorname{Var}_{0} Z_{t-i}}}+\sqrt{\frac{\operatorname{Var}_{1} Z_{t-i} / \operatorname{Var}_{0} Z_{t-i}}{\operatorname{Var}_{1} Z_{t} / \operatorname{Var}_{0} Z_{t}}} .
$$

Since the right side of inequality (2.2) is always greater or equal to 2 it follows that $\operatorname{Corr}_{1}\left(Z_{t}, Z_{t-i}\right) \geq \operatorname{Corr}_{0}\left(Z_{t}, Z_{t-i}\right)$. If the correlation is nondecreasing then inequality (2.2) gives a stronger lower bound. In order to prove monotonicity it has to be shown more.

Remarks 2.2 1. The inequality (2.2) can be written as

$$
2 \frac{\operatorname{Cov}_{1}\left(Z_{t}, Z_{t-i}\right)}{\operatorname{Cov}_{0}\left(Z_{t}, Z_{t-i}\right)} \geq \frac{\operatorname{Var}_{1} Z_{t}}{\operatorname{Var}_{0} Z_{t}}+\frac{\operatorname{Var}_{1} Z_{t-i}}{\operatorname{Var}_{0} Z_{t-i}} .
$$

2. Condition (2.1) is very weak. Let $\Gamma=\left(\gamma_{v \mu}\right), \Delta=\left(\delta_{v \mu}\right)$, and $\mathbf{w}_{t}=\left(w_{t v}\right)$. Suppose that there is $v \in\{1, \ldots, t-i\}$ and $\mu \in\{1, \ldots, t\}$ such that $\gamma_{\nu \mu}>\delta_{\nu \mu}$. Then (2.1) holds if $w_{t \mu} w_{t-i, v}>0$. Note that for $\Gamma_{t}=\Delta_{t}$ the theorem is trivial.

Next it is assumed that the covariance matrix of $\mathbf{X}_{t}$ is a Toeplitz matrix, i.e. it is of the type $\operatorname{Cov}\left(\mathbf{X}_{t}\right)=\left(\gamma_{|i-j|}\right)$. Using the matrices $U_{v t}=\left(I_{\{v\}}(|j-\ell|)\right)_{j, \ell=1, \ldots, t}$ it follows that $\operatorname{Cov}\left(\mathbf{X}_{t}\right)=\sum_{v=0}^{t-1} \gamma_{\nu} U_{v t}$. In the following $I_{A}(t)$ denotes the indicator function of a set $A$, e.g. it is equal to 1 if $t \in A$, else to 0 . Furthermore we get

$$
\begin{aligned}
& \operatorname{Var}\left(Z_{t}\right)=\frac{\gamma_{0}}{2} W_{0 t}+\sum_{\nu=1}^{t-1} \gamma_{\nu} W_{\nu t}, \quad \operatorname{Cov}\left(Z_{t}, Z_{t-i}\right)=\frac{\gamma_{0}}{2} W_{0 t i}+\sum_{\nu=1}^{t-1} \gamma_{\nu} W_{\nu t i} \quad \text { with } \\
& W_{v t}=\mathbf{w}_{t}^{\prime} U_{v t} \mathbf{w}_{t}, \quad W_{v t i}=\mathbf{w}_{t}^{\prime} U_{v t} \tilde{\mathbf{w}}_{t} \quad \text { for } \quad v=1, \ldots, t-1, \\
& W_{0 t}=2 \mathbf{w}_{t}^{\prime} U_{0 t} \mathbf{w}_{t}, \quad W_{0 t i}=2 \mathbf{w}_{t}^{\prime} U_{0 t} \tilde{\mathbf{w}}_{t} .
\end{aligned}
$$

Moreover, we define $W_{v, t-i}=0$ for $t-i \leq v \leq t-1$. On the first view the use of the factor 2 in the definitions of $W_{0 t}$ and $W_{0 t i}$ seems to be artificial. Nevertheless, this leads to more elegant representations and simplifies distinguishing between special cases (see (2.8), Sections 3.2.1, 4.2, 4.3). 
Theorem 2.3 Let $\Delta_{t}=\left(\delta_{|v-j|}\right)$ and $\Gamma_{t}=\left(\gamma_{|v-j|}\right)$ be covariance matrices of Toeplitz type. Assume that

$$
\begin{array}{lll}
\gamma_{0} \delta_{0}>0, \gamma_{h} \geq 0, \delta_{h} \geq 0 & \text { for } & 1 \leq h \leq t-1 \\
\gamma_{\ell} \delta_{j} \geq \gamma_{j} \delta_{\ell} & \text { for all } & 0 \leq j<\ell \leq t-1 .
\end{array}
$$

Let $i \in\{0, \ldots, t-1\}$ be fixed. For the weights it is demanded that for all $0 \leq j<\ell \leq t-1$ and all $0 \leq v \leq t-1$

$$
W_{\ell t i}\left(W_{j t} W_{\nu, t-i}+W_{v t} W_{j, t-i}\right)-W_{j t i}\left(W_{\ell t} W_{\nu, t-i}+W_{v t} W_{\ell, t-i}\right) \geq 0 .
$$

Then the inequality (2.2) holds.

The next result is an immediate consequence of Theorem 2.3 and Slepian's inequality.

Corollary 2.4 Assume that the conditions of Theorem 2.1 and Theorem 2.3 are fulfilled for all $t \in\{1, \ldots, k\}$ and for all $i \in\{0, \ldots, t-1\}$ then it holds that for any real numbers $c_{1}, \ldots, c_{k}$

$$
P_{\tau}\left(\mathbf{w}_{t}^{\prime} \mathbf{X}_{t} \leq c_{t} \sqrt{\mathbf{w}_{t}^{\prime} C_{k}(\tau) \mathbf{w}_{t}}, t=1, \ldots, k\right)
$$

is a nondecreasing function in $\tau$.

Now let $N_{\tau}=\inf \left\{t \in \mathbb{N}: Z_{t}>c \sqrt{\operatorname{Var}_{\tau}\left(Z_{t}\right)}\right\}$ denote the run length of the control chart based on $Z_{t}$. It is equal to the first value at which the process is concluded to be out-ofcontrol. This means that its expectation is greater than zero. The Corollary 2.4 says that the tail probability $P_{\tau}\left(N_{\tau}>k\right)$ is nondecreasing in $\tau$. This is an extension of the results of [9] and [10]. In these papers the EWMA (exponentially weighted moving average) chart is analyzed while here a very general family of control schemes is considered.

Next we want to discuss the conditions of Theorem 2.3. The assumptions (2.4) and (2.5) are conditions on the autocovariances of the underlying process. These assumptions are the same as in [10]. If $\delta_{v}>0$ for all $0 \leq v \leq t-1$, they are equivalent to demanding that $\left\{\gamma_{\nu} / \delta_{\nu}\right\}$ is nondecreasing in $v$. It is proved in [10] that it is not sufficient to assume that the autocorrelation of the target process is nondecreasing. Assumption (2.6) limits the choice of the weights. Next, sufficient conditions for the validity of (2.6) are derived which are fulfilled for most weight functions arising in practice.

In many cases of interest the weights $\mathbf{w}_{t}^{\prime}=\left(w_{t 1}, \ldots, w_{t t}\right)$ are of the type $w_{t j}=$ $K(t-j), j=1, \ldots, t$ with a nonnegative function $K$. Thus $Z_{t}=\sum_{j=1}^{t} K(t-j) X_{j}$. We denote the corresponding charts as kernel charts. Several examples will be discussed in Section 3. We get with $\kappa_{\nu t}=\sum_{j=0}^{t-|\nu|} K(j) K(j+|\nu|)$ that $W_{\nu t}=2 \kappa_{\nu, t-1}$ and

$$
W_{v t i}=\kappa_{i-v, t-1-\min \{i, \nu\}}+\kappa_{i+v, t-1}=\left(W_{i-v, t-\min \{i, v\}}+W_{i+v, t}\right) / 2 .
$$

Note that $\kappa_{v t}$ is equal to the autocovariance at lag $v$ of an $\mathrm{MA}(t)$ process. 
Proposition 2.5 a) Suppose that there is $m \in\{1, \ldots, t-i-1\}$ such that $K(0) \geq \cdots \geq$ $K(m-1)>K(m)=\cdots=K(t-1)=0$. Then the inequality (2.6) holds for all $0 \leq j<\ell \leq t-1$ and all $0 \leq v \leq t-1$ if

$$
\frac{W_{j+1, t i}}{W_{j+1, t}} \geq \frac{W_{j t i}}{W_{j t}} \quad \text { for all } \quad 0 \leq j \leq m-2 .
$$

b) Let $v \in\{0, \ldots, t-1\}$ be fixed. Then the inequality (2.6) is satisfied for all $0 \leq j<$ $\ell \leq t-1$ if the following conditions $i)$ - iii) are fulfilled:

i) $K(0) \geq \cdots \geq K(t-1)>0$

ii) $K(j) K(j+2) \geq K(j+1)^{2}$ for $0 \leq j \leq t-3$

iii) $\frac{W_{j+1, t i}}{W_{j+1, t}} \geq \frac{W_{j t i}}{W_{j t}}$ for all $0 \leq j \leq t-2$.

c) Let $i, v \in\{0, \ldots, t-1\}$ be fixed. Suppose that condition i) of part b) holds. Then (2.6) is satisfied for all $0 \leq j<\ell \leq t-1$ if

$$
\frac{W_{j t i}}{W_{j t} W_{\nu, t-i}+W_{\nu t} W_{j, t-i}} \quad \text { is nondecreasing in } \quad j \in\{0, \ldots, t-1\} \text {. }
$$

\section{Examples}

In this section let $\mathbf{X}_{t}$ be normally distributed with mean 0 and with a covariance matrix of Toeplitz type. All of the following charts can be considered as kernel charts. It is shown that the conditions of Theorem 2.1 and Theorem 2.3 are fulfilled for many control charts provided that the smoothing parameters satisfy certain restrictions. The proofs of all results are given in the appendix.

\subsection{The moving average chart with constant weights}

This control scheme is based on the simple idea to smooth the last $m$ observations by averaging. If at least $m$ observations are available then each value gets the same weight. Else, the weight is equal to the number of observed data. Consequently we obtain that for $t \geq 1$

$$
Z_{t}=\sum_{j=\max \{t+1-m, 1\}}^{t} X_{j} / \min \{t, m\}
$$

Because the denominator can be neglected (see appendix) a kernel chart is presented with $K(j)=I_{\{0, \ldots, m-1\}}(j)$.

Proposition 3.1 Let $N_{\tau}$ be the run length of the control scheme based on $\left\{Z_{t}\right\}$ given in (3.1). Then $P_{\tau}\left(N_{\tau}>k\right)$ is a nondecreasing function in $\tau$. 


\subsection{The ARMA chart}

The ARMA control chart is a relatively new control scheme. It was introduced in [3]. The idea of the chart is to transform the original time series to a new one such that a particular shift can be detected faster.

Their control statistic $Z_{t}^{*}$ is the causal solution of the ARMA process given by $P(B) Z_{t}^{*}=Q(B) X_{t}$, where $B$ stands for the backward shift operator, i.e. $B X_{t}=X_{t-1}$. Furthermore let $P(z)=1-\sum_{j=1}^{p} \alpha_{j} z^{j}$ and $Q(z)=\sum_{j=0}^{q} \beta_{j} z^{j}$. In [3] recommendations are given how the design parameters should be chosen. They focus on $\operatorname{ARMA}(1,1)$ processes.

If the control statistic is applied in practice a starting problem arises because the observations for $t \leq 0$ are not known. This problem is not considered in [3]. This simplification facilitates many things. Theoretical statements are easier to derive since $\left\{Z_{t}^{*}\right\}$ is a stationary process. However, we think that this is an unrealistic assumption. For that reason we choose another way. Now $\left\{Z_{t}^{*}\right\}$ can be written as $Z_{t}^{*}=\sum_{j=0}^{\infty} \Psi_{j} X_{t-j}$. Truncating the sum we get our control statistic $Z_{t}=\sum_{j=0}^{t-1} \Psi_{j} X_{t-j}$. Therefore $K(j)=$ $\Psi_{j}$. Note that $\left\{Z_{t}\right\}$ is not stationary.

\subsubsection{The moving average chart with variable weights}

First, we deal with the case $p=0$. Then the control statistic is equal to

$$
Z_{t}=\sum_{j=0}^{\min \{t-1, q\}} \beta_{j} X_{t-j}, \quad t \geq 1 .
$$

The kernel is given by $K(j)=\beta_{j} I_{\{0, \ldots, q\}}(j)$. Since the weights are assumed to be nonnegative we demand that $\beta_{0} \geq 0, \ldots, \beta_{q} \geq 0$. We get that (cf. (2.8))

$$
\begin{aligned}
W_{\nu t i} & =\sum_{j=0}^{\min \{t-1-\min \{i, v\}, q\}-|i-v|} \beta_{j} \beta_{j+|i-v|}+\sum_{j=0}^{\min \{t-1, q\}-i-v} \beta_{j} \beta_{j+i+v} \\
& =\kappa_{i-v, \min \{t-1-\min \{i, v\}, q\}}+\kappa_{i+v, \min \{t-1, q\} .}
\end{aligned}
$$

Consequently it follows that

$$
W_{v t i}= \begin{cases}\kappa_{i-v, t-1-\min \{i, v\}}+\kappa_{i+v, t-1} & \text { for } t \leq q \\ \kappa_{i-v, t-1-\min \{i, v\}}+\kappa_{i+v, q} & \text { for } q+1 \leq t \leq q+\min \{i, v\} \\ \kappa_{\nu-i, q}+\kappa_{v+i, q} & \text { for } t \geq q+1+\min \{i, v\} .\end{cases}
$$

Let $t \geq q+i+1$ and let $0<\beta_{q} \leq \cdots \leq \beta_{0}$. Then Proposition 2.5a) shows that condition (2.6) is satisfied if

$$
\kappa_{j+1, q}\left(\kappa_{i+j, q}+\kappa_{j-i, q}\right) \leq \kappa_{j q}\left(\kappa_{i+j+1, q}+\kappa_{j+1-i, q}\right)
$$

for all $0 \leq j \leq q-1$. For small values of $q$ condition (2.6) can be checked directly.

Proposition 3.2 Let $N_{\tau}$ be the run length of the control scheme based on $\left\{Z_{t}\right\}$ given in (3.2). Suppose that $q=2,0 \leq \sqrt{\beta_{2} / \beta_{0}} \leq \beta_{1} / \beta_{0} \leq 1$ and that $\beta_{2} \leq \beta_{1}$. Then $P_{\tau}\left(N_{\tau}>k\right)$ is a nondecreasing function in $\tau$. 


\subsubsection{The EWMA chart}

The well-known EWMA chart with starting value $Z_{0}=0$ can be considered as an $\operatorname{ARMA}(1,0)$ chart. It can be obtained by setting $\alpha_{1}=1-\lambda$ and $\beta_{0}=\lambda$. The weights are given by $w_{t j}=\lambda(1-\lambda)^{t-j}$, i.e. $K(j)=\lambda(1-\lambda)^{j}$. The Corollary 2.4 was proved for the EWMA chart in [10]. They made use of a different type of proof which is completely geared to exponential weights. It cannot be generalized to more general structures.

\subsubsection{The ARMA(1,1) chart}

Let $p=q=1$ and $\left|\alpha_{1}\right|<1$. A stationary $\operatorname{ARMA}(1,1)$ process can be written as

$$
Z_{t}^{*}=\beta_{0} X_{t}+\sum_{j=1}^{\infty}\left(\alpha_{1}^{j} \beta_{0}+\alpha_{1}^{j-1} \beta_{1}\right) X_{t-j}
$$

Our control statistic is obtained by truncating the series. This leads to

$$
Z_{t}=\sum_{j=0}^{t-1} \Psi_{j} X_{t-j} \quad \text { with } \quad \Psi_{0}=\beta_{0}, \Psi_{j}=\alpha_{1}^{j-1}\left(\beta_{0} \alpha_{1}+\beta_{1}\right) \quad \text { for } \quad j \geq 1
$$

Proposition 3.3 Let $N_{\tau}$ be the run length of the control scheme based on $\left\{Z_{t}\right\}$ given in (3.4). Suppose that $0 \leq \alpha_{1}<1, \beta_{1} \geq 0$ and $\beta_{0} \geq \beta_{1} /\left(1-\alpha_{1}\right)$. Then $P_{\tau}\left(N_{\tau}>k\right)$ is a nondecreasing function in $\tau$.

Note that Proposition 3.3 includes the EWMA chart. In Figure 1 the probability of no signal up to time $k$ is shown for the $\operatorname{ARMA}(1,1)$ chart $Z_{t}=0.5 Z_{t-1}+X_{t}+0.4 X_{t-1}$. The covariance matrices $\Delta_{k}$ and $\Gamma_{k}$ of the $\left\{X_{t}\right\}$ are covariance matrices of an $\operatorname{AR}(1)$ process with parameter 0 and 0.99 , respectively. Thus $C_{k}(\tau)=\left((1-\tau) I_{\{0\}}(|i-j|)+\right.$ $\left.\tau 0.99^{|i-j|} /\left(1-0.99^{2}\right)\right)_{i, j=1, \ldots, k}$. Here $P_{\tau}\left(N_{\tau}>k\right)$ is estimated via a simulation study based on $10^{8}$ repetitions. The figure illustrates that the probability of no signal takes the smallest value at $\tau=0$, e.g. if the variables $\left\{X_{t}\right\}$ are independent. The probability increases if $\tau$ increases.

\section{Appendix}

\subsection{Proofs of Theorem 2.1 and Theorem 2.3}

Proof of Theorem 2.1: We obtain that

$$
\begin{aligned}
& \frac{\partial \operatorname{Corr}_{\tau}\left(Z_{t}, Z_{t-i}\right)^{2}}{\partial \tau} \\
& =\frac{\operatorname{Corr}_{\tau}\left(Z_{t}, Z_{t-i}\right)}{\left(\operatorname{Var}_{\tau} Z_{t} \operatorname{Var}_{\tau} Z_{t-i}\right)^{3 / 2}}\left\{2 \operatorname{Var}_{\tau} Z_{t} \operatorname{Var}_{\tau} Z_{t-i} \frac{\partial \operatorname{Cov}_{\tau}\left(Z_{t}, Z_{t-i}\right)}{\partial \tau}\right. \\
& \left.-\operatorname{Cov}_{\tau}\left(Z_{t}, Z_{t-i}\right)\left(\operatorname{Var}_{\tau} Z_{t} \frac{\partial \operatorname{Var}_{\tau} Z_{t-i}}{\partial \tau}+\operatorname{Var}_{\tau} Z_{t-i} \frac{\partial \operatorname{Var}_{\tau} Z_{t}}{\partial \tau}\right)\right\} .
\end{aligned}
$$




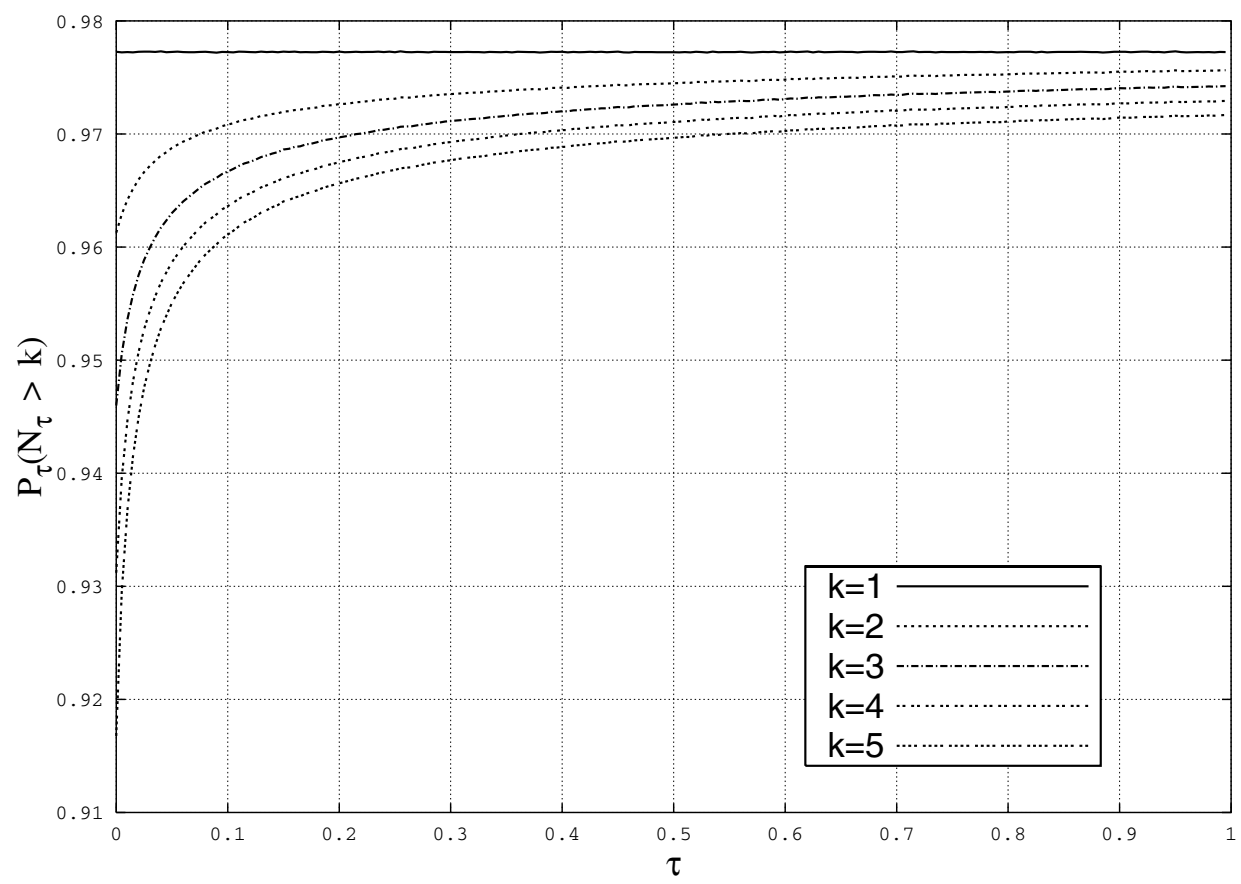

Figure 1: Plot of $P_{\tau}\left(N_{\tau}>k\right)$ as a function of $\tau$ and $k$ for the $\operatorname{ARMA}(1,1)$ chart with $\alpha_{1}=0.5$, $\beta_{0}=1, \beta_{1}=0.4, c=2.0\left(\delta_{0}=1, \delta_{i}=0\right.$ for $i \geq 1$ and $\gamma_{i}=0.99^{i} /\left(1-0.99^{2}\right)$ for $\left.i \geq 0\right)$.

Consequently $\operatorname{Corr}_{\tau}\left(Z_{t}, Z_{t-i}\right)$ is a nondecreasing function in $\tau$ if and only if

$$
2 \frac{\frac{\partial \operatorname{Cov}_{\tau}\left(Z_{t}, Z_{t-i}\right)}{\partial \tau}}{\operatorname{Cov}_{\tau}\left(Z_{t}, Z_{t-i}\right)} \geq \frac{\frac{\partial \operatorname{Var}_{\tau} Z_{t}}{\partial \tau}}{\operatorname{Var}_{\tau} Z_{t}}+\frac{\frac{\partial \operatorname{Var}_{\tau} Z_{t-i}}{\partial \tau}}{\operatorname{Var}_{\tau} Z_{t-i}}
$$

Note that $\operatorname{Cov}_{\tau}\left(Z_{t}, Z_{t-i}\right)>0$ for any value of $\tau$. Since $\operatorname{Cov}_{\tau}\left(Z_{t}, Z_{t-i}\right)=\mathbf{w}_{t}^{\prime}((1-$ $\left.\tau) \Delta_{t}+\tau \Gamma_{t}\right) \tilde{\mathbf{w}}_{t}$ we get that the inequality (4.2) can be written as follows

$$
2 \frac{1}{\tau+\frac{\mathbf{w}_{t}^{\prime} \Delta_{t} \tilde{\mathbf{w}}_{t}}{\mathbf{w}_{t}^{\prime}\left(\Gamma_{t}-\Delta_{t}\right) \tilde{\mathbf{w}}_{t}}} \geq \frac{1}{\tau+\frac{\mathbf{w}_{t}^{\prime} \Delta_{t} \mathbf{w}_{t}}{\mathbf{w}_{t}^{\prime}\left(\Gamma_{t}-\Delta_{t}\right) \mathbf{w}_{t}}}+\frac{1}{\tau+\frac{\tilde{\mathbf{w}}_{t}^{\prime} \Delta_{t} \tilde{\mathbf{w}}_{t}}{\tilde{\mathbf{w}}_{t}^{\prime}\left(\Gamma_{t}-\Delta_{t}\right) \tilde{\mathbf{w}}_{t}}} .
$$

(4.3) is of the type $2 /(\tau+c)-1 /(\tau+a)-1 /(\tau+b) \geq 0$ for all $0 \leq \tau \leq 1$ with $a>0, b>0$ and $c>0$. But this is valid if and only if it holds for $\tau=0$, i.e. if and only if $2 / c \geq 1 / a+1 / b$. This implies that (4.3) is equivalent to

$$
2 \frac{\mathbf{w}_{t}^{\prime} \Gamma_{t} \tilde{\mathbf{w}}_{t}}{\mathbf{w}_{t}^{\prime} \Delta_{t} \tilde{\mathbf{w}}_{t}} \geq \frac{\mathbf{w}_{t}^{\prime} \Gamma_{t} \mathbf{w}_{\mathbf{t}}}{\mathbf{w}_{t}^{\prime} \Delta_{t} \mathbf{w}_{t}}+\frac{\tilde{\mathbf{w}}_{t}^{\prime} \Gamma_{t} \tilde{\mathbf{w}}_{t}}{\tilde{\mathbf{w}}_{t}^{\prime} \Delta_{t} \tilde{\mathbf{w}}_{t}} .
$$

But this is only another representation of (2.3). 
Proof of Theorem 2.3: According to the definition of $U_{v t}$, the covariance matrices $\Gamma_{t}$ and $\Delta_{t}$ can we rewritten as $\Gamma_{t}=\sum_{\nu=0}^{t-1} \gamma_{\nu} U_{v t}$ and $\Delta_{t}=\sum_{\nu=0}^{t-1} \delta_{\nu} U_{v t}$. Let $\gamma_{j}^{*}=\gamma_{j}$ and $\delta_{j}^{*}=\delta_{j}$ for $j=1, \ldots, t-1, \gamma_{0}^{*}=\gamma_{0} / 2, \delta_{0}^{*}=\delta_{0} / 2$. Using these presentations we see that (2.3) is equivalent to

$$
2 \frac{\sum_{\nu=0}^{t-1} \gamma_{\nu}^{*} W_{v t i}}{\sum_{\nu=0}^{t-1} \delta_{v}^{*} W_{v t i}} \geq \frac{\sum_{\nu=0}^{t-1} \gamma_{\nu}^{*} W_{\nu t}}{\sum_{\nu=0}^{t-1} \delta_{v}^{*} W_{v t}}+\frac{\sum_{\nu=0}^{t-1} \gamma_{\nu}^{*} W_{\nu, t-i}}{\sum_{\nu=0}^{t-1} \delta_{\nu}^{*} W_{\nu, t-i}}
$$

Because all denominators are positive this is equivalent to

$$
\begin{aligned}
& \sum_{\nu_{1}, \nu_{2}, \nu_{3}=0}^{t-1} \gamma_{\nu_{1}}^{*} W_{\nu_{1} t i} \delta_{\nu_{2}}^{*} W_{\nu_{2} t} \delta_{\nu_{3}}^{*} W_{\nu_{3}, t-i}-\sum_{\nu_{1}, \nu_{2}, \nu_{3}=0}^{t-1} \delta_{\nu_{1}}^{*} W_{\nu_{1} t i} \gamma_{\nu_{2}}^{*} W_{\nu_{2} t} \delta_{\nu_{3}}^{*} W_{\nu_{3}, t-i} \\
& +\sum_{\nu_{1}, \nu_{2}, \nu_{3}=0}^{t-1} \gamma_{\nu_{1}}^{*} W_{\nu_{1} t i} \delta_{\nu_{2}}^{*} W_{\nu_{2} t} \delta_{\nu_{3}}^{*} W_{\nu_{3}, t-i}-\sum_{\nu_{1}, \nu_{2}, \nu_{3}=0}^{t-1} \delta_{v_{1}}^{*} W_{\nu_{1} t i} \delta_{\nu_{2}}^{*} W_{\nu_{2} t} \gamma_{\nu_{3}}^{*} W_{\nu_{3}, t-i} \geq 0 .
\end{aligned}
$$

Swapping the indices $v_{2}$ and $v_{3}$ in the third and fourth terms we get

$$
\begin{aligned}
& \sum_{\nu_{1}, \nu_{2}, \nu_{3}=0}^{t-1} \delta_{v_{3}}^{*}\left(\gamma_{\nu_{1}}^{*} \delta_{\nu_{2}}^{*}-\gamma_{\nu_{2}}^{*} \delta_{\nu_{1}}^{*}\right) W_{\nu_{1} t i} W_{\nu_{2} t} W_{\nu_{3}, t-i} \\
& +\sum_{\nu_{1}, \nu_{2}, \nu_{3}=0}^{t-1} \gamma_{\nu_{1}}^{*} W_{\nu_{1} t i} \delta_{v_{3}}^{*} W_{\nu_{3} t} \delta_{\nu_{2}}^{*} W_{\nu_{2}, t-i}-\sum_{\nu_{1}, \nu_{2}, \nu_{3}=0}^{t-1} \delta_{\nu_{1}}^{*} W_{\nu_{1} t i} \delta_{\nu_{3}}^{*} W_{\nu_{3} t} \gamma_{\nu_{2}}^{*} W_{\nu_{2}, t-i} \geq 0 .
\end{aligned}
$$

Consequently (2.3) can be rewritten in the form

$$
\sum_{\nu_{1}, \nu_{2}, \nu_{3}=0}^{t-1}\left(\gamma_{\nu_{1}}^{*} \delta_{\nu_{2}}^{*}-\gamma_{\nu_{2}}^{*} \delta_{\nu_{1}}^{*}\right) \delta_{v_{3}}^{*}\left(W_{\nu_{1} t i} W_{\nu_{2} t} W_{\nu_{3}, t-i}+W_{\nu_{1} t i} W_{\nu_{3} t} W_{\nu_{2}, t-i}\right) \geq 0
$$

According to the assumptions of the theorem $\gamma_{\nu_{1}}^{*} \delta_{\nu_{2}}^{*}-\gamma_{\nu_{2}}^{*} \delta_{v_{1}}^{*} \geq 0$ and $\gamma_{\nu_{2}}^{*} \delta_{v_{1}}^{*}-\gamma_{\nu_{1}}^{*} \delta_{v_{2}}^{*} \leq 0$ for $0 \leq v_{2}<v_{1} \leq t-1$. Since both terms are present in (4.4) a sufficient condition for the sum to be nonnegative is that for all $0 \leq j<\ell \leq t-1$ and all $0 \leq v \leq t-1$

$$
\begin{aligned}
& W_{\ell t i}\left(W_{j t} W_{\nu, t-i}+W_{\nu t} W_{j, t-i}\right)-W_{j t i}\left(W_{\ell t} W_{\nu, t-i}+W_{\nu t} W_{\ell, t-i}\right) \\
& \quad=W_{\nu, t-i}\left(W_{\ell t i} W_{j t}-W_{j t i} W_{\ell t}\right)+W_{v t}\left(W_{\ell t i} W_{j, t-i}-W_{j t i} W_{\ell, t-i}\right) \geq 0 .
\end{aligned}
$$

Proof of Proposition 2.5: a) We get that $W_{j t}=W_{j, t-i}>0$ for all $j \in\{0, \ldots, m-1\}$ and $W_{j t}=W_{j, t-i}=0$ for all $j \in\{m, \ldots, t-1\}$. Then the inequality (2.6) holds for all $0 \leq j<\ell \leq t-1$ and all $0 \leq v \leq t-1$ if

$$
\frac{W_{j t i}}{W_{j t}} \leq \frac{W_{j+1, t i}}{W_{j+1, t}} \quad \text { for all } \quad 0 \leq j \leq m-2
$$


b) First we observe that $w_{t j} \leq w_{t, j+1}$ for $1 \leq j \leq t-1$ and $W_{j t} \geq W_{j, t-i}>0$. Then (2.6) holds if

$$
W_{j+1, t i}\left(W_{j t}+W_{j, t-i}\right) \geq W_{j t i}\left(W_{j+1, t}+W_{j+1, t-i}\right)
$$

for all $0 \leq j \leq t-2$, and

$$
W_{j+1, t i} W_{j, t-i} \geq W_{j t i} W_{j+1, t-i} \text { for all } 0 \leq j \leq t-i-1 .
$$

We shall show that (4.6) and (4.7) are valid. This will prove part c) of the proposition. We obtain for $0 \leq j \leq t-i-1$

$$
\begin{gathered}
I:=\sum_{v=t-i-j-1}^{\substack{t-j-2 \\
t-j-2}} \sum_{\mu=0}^{t-i-j-2} K(v) K(\mu)(K(v+j+1) K(\mu+j)-K(\mu+j+1) K(v+j)), \\
I I:=\sum_{\mu=0}^{t-j+1, t} K(\mu) K(\mu+j+1) K(t-i-j-1) K(t-i-1) \\
-\sum_{\mu=0}^{t-i-j-2} K(\mu) K(\mu+j+1) K(t-1-j) K(t-1) .
\end{gathered}
$$

Since $K(v)$ is decreasing it follows that $I I \geq 0$. Using condition ii) we get that $K(v+$ $j+1) / K(v+j) \geq K(\mu+j+1) / K(\mu+j)$ for $v>\mu$. Consequently it holds that

$$
\frac{W_{j+1, t}}{W_{j t}} \geq \frac{W_{j+1, t-i}}{W_{j, t-i}} \text { for all } 0 \leq j \leq t-i-1 .
$$

If condition iii) and (4.8) are valid then (4.7) follows because

$$
\frac{W_{j+1, t i}}{W_{j t i}} \geq \frac{W_{j+1, t}}{W_{j t}} \geq \frac{W_{j+1, t-i}}{W_{j, t-i}} .
$$

Because of iii) and (4.7) the condition (4.6) is fulfilled, too.

\subsection{Proofs of Proposition 3.1 and Proposition 3.3}

Proof of Proposition 3.1: Let $\tilde{Z}_{t}=\sum_{j=\max \{t+1-m, 1\}}^{t} X_{j}$ then

$$
P_{\tau}\left(Z_{t} \leq c_{t} \sqrt{\operatorname{Var}_{\tau}\left(Z_{t}\right)}, t=1, \ldots, k\right)=P_{\tau}\left(\tilde{Z}_{t} \leq c_{t} \sqrt{\operatorname{Var}_{\tau}\left(\tilde{Z}_{t}\right)}, t=1, \ldots, k\right) .
$$

Therefore we work with $\tilde{Z}_{t}$ instead of $Z_{t}$.

Case I: Suppose that $t \geq m+i$. Then we get that for $i \geq m$

$$
W_{v t i}=\left\{\begin{array}{cl}
m-|i-v| & \text { for } i-m+1 \leq v \leq i+m-1 \\
0 & \text { for } 0 \leq v \leq i-m \text { or } i+m \leq v \leq t-1
\end{array}\right.
$$


while for $i \leq m-1$ it holds that

$$
W_{v t i}=\left\{\begin{array}{cll}
2(m-\max \{i, v\}) & \text { for } \quad 0 \leq v \leq m-i-1 \\
m-|i-v| & \text { for } \quad m-i \leq v \leq m+i-1 \\
0 & \text { for } \quad m+i \leq v \leq t-1
\end{array}\right.
$$

Putting $i=0$ we get that $W_{v t}=2(m-v) I_{\{0, \ldots, m-1\}}(v)$ for $t \geq m$. Because of Proposition $2.5 \mathrm{a}$ ) condition (2.6) is satisfied if

$$
W_{j+1, t i} W_{j t}-W_{j t i} W_{j+1, t} \geq 0 \text { for } 0 \leq j \leq m-2 .
$$

First we deal with the case $i \geq m$. Since $W_{j t i}$ is not equal to zero for $i-m+1 \leq j \leq$ $i+m-1$ it is sufficient to discuss the behaviour for $i-m+1 \leq j \leq m-2$. Then $W_{j t i}=m-i+j$. Since $(m-i+j+1)(m-j)-(m-i+j)(m-j-1) \geq 0$ the condition (2.6) is satisfied.

Next let $i \leq m-1$. For $i \leq m-i-1$ we obtain

$$
W_{j t i}=\left\{\begin{array}{cl}
2(m-i) & \text { for } \quad 0 \leq j \leq i \\
2(m-j) & \text { for } i+1 \leq j \leq m-i-1 \\
m-j+i & \text { for } \quad m-i \leq j \leq m+i-1
\end{array}\right.
$$

while for $i>m-i-1$

$$
W_{j t i}=\left\{\begin{array}{cl}
2(m-i) & \text { for } \quad 0 \leq j \leq m-i-1 \\
m-i+j & \text { for } \quad m-i \leq j \leq i \\
m-j+i & \text { for } i<j \leq m+i-1
\end{array}\right.
$$

Distinguishing between these cases it can easily be proved that (4.9) holds.

Case II: The cases $m \leq t \leq m+i-1$ and $1 \leq t \leq m-1$ are treated by analogy.

Proof of Proposition 3.3: Because the proof is based on long and tedious calculations we only sketch the main ideas. The complete proof is given in [8].

Let $\gamma=\frac{\beta_{0} \alpha_{1}}{\alpha_{1} \beta_{0}+\beta_{1}}$ and $\tilde{X}_{t}=\left(\alpha_{1} \beta_{0}+\beta_{1}\right) X_{t} / \alpha_{1}$. This shows that $Z_{t}=\gamma \tilde{X}_{t}+$ $\sum_{j=1}^{t-1} \alpha_{1}^{j} \tilde{X}_{t-j}$. Here it is assumed that $\alpha_{1}>0$. This is no restriction since the case $\alpha_{1}=0$ was already discussed in Section 3.2.1. Because $\tilde{X}_{t}$ is again a stationary process we can restrict ourselves to this representation. Consequently $K(0)=\gamma$ and $K(j)=\alpha_{1}^{j}$ for $1 \leq j \leq t-1$. $K$ is nonincreasing since $\beta_{0} \geq \beta_{1} /\left(1-\alpha_{1}\right)$. For the reasons of simplicity we make use of the easier notation $Z_{t}=\gamma X_{t}+\sum_{j=1}^{t-1} \alpha_{1}^{j} X_{t-j}$ with $\alpha_{1}>0$ and $\gamma>0$. We get for $0 \leq v \leq t$

$$
\kappa_{\nu t}=\gamma K(v)+\alpha_{1}^{\nu+2} \frac{1-\alpha_{1}^{2(t-v)}}{1-\alpha_{1}^{2}}=A \alpha_{1}^{v}\left(K^{*}(v)-\alpha_{1}^{2(t-v)}\right)
$$

with $K^{*}(j)=\frac{\gamma}{A}+1=$ : $\gamma_{1}$ for $j \geq 1, K^{*}(0)=\frac{\gamma^{2}}{A}+1=$ : $\gamma_{2}$ and $A=\alpha_{1}^{2} /\left(1-\alpha_{1}^{2}\right)$. Using (2.8) it follows that for $t-i \leq v \leq t-1$

$W_{v t i}=A \alpha_{1}^{|i-v|}\left(K^{*}(|i-v|)-\alpha_{1}^{2(t-1-\max \{i, \nu\})}\right)=\left\{\begin{array}{cl}\alpha_{1}^{-\min \{v, i\}} \kappa_{\max \{v, i\}, t-1} & \text { for } i \neq v \\ \kappa_{0, t-i-1} & \text { for } i=v\end{array}\right.$ 
and that for $0 \leq v \leq t-i-1$

$$
W_{v t i}=\left\{\begin{array}{cc}
\alpha_{1}^{-\min \{v, i\}} \kappa_{\max \{v, i\}, t-1}+\alpha_{1}^{i} \kappa_{v, t-i-1} & \text { for } i \neq v \\
\kappa_{0, t-i-1}+\alpha_{1}^{i} \kappa_{i, t-i-1} & \text { for } i=v
\end{array}\right.
$$

Furthermore $W_{v t}=2 \kappa_{v, t-1}$.

For further verification we need some properties of $\kappa_{v t}$. It can immediately be seen that $\alpha_{1}^{-1} \kappa_{j, t}-\kappa_{j+1, t} \geq 0$ for $j \geq 0$ and

$$
\kappa_{0, t-i-1}=\alpha_{1}^{-i} \kappa_{i, t-1}+A\left(K^{*}(0)-K^{*}(i)\right) \text { for } i \geq 0 .
$$

It is necessary to distinguish between several cases. We describe the more complicate case I in more detail while the proof of the remaining parts is omitted. The interested reader is referred to [8].

Case I: Let $v \leq t-i-1$ and $j+1 \leq t-i-1$

We make use of Proposition 2.5c). It is sufficient to prove that

$I I I:=\left(W_{j+1, t i}\left(W_{j t} W_{v, t-i}+W_{v t} W_{j, t-i}\right)-W_{j t i}\left(W_{j+1, t} W_{v, t-i}+W_{v t} W_{j+1, t-i}\right)\right) / 4 \geq 0$.

a) Let $j+1 \neq i$ and $j \neq i$. Then

$$
\begin{aligned}
& I I I=\alpha_{1}^{-\min \{j+1, i\}} \kappa_{\max \{j+1, i\}, t-1}\left(\kappa_{j, t-1} \kappa_{\nu, t-i-1}+\kappa_{\nu, t-1} \kappa_{j, t-i-1}\right) \\
&-\alpha_{1}^{-\min \{j, i\}} \kappa_{\max \{j, i\}, t-1}\left(\kappa_{j+1, t-1} \kappa_{v, t-i-1}+\kappa_{\nu, t-1} \kappa_{j+1, t-i-1}\right) \\
&+\alpha_{1}^{i} \kappa_{\nu, t-i-1}\left(\kappa_{j+1, t-i-1} \kappa_{j, t-1}-\kappa_{j, t-i-1} \kappa_{j+1, t-1}\right) .
\end{aligned}
$$

$\left.a_{1}\right)$ Let $i<j$ then we get using $\left|\alpha_{1}\right|<1$ and $\gamma \geq \alpha_{1}$

$$
\begin{array}{r}
I I I=\left(\kappa_{j, t}-i-1 \kappa_{j+1, t-1}-\kappa_{j+1, t-i-1} \kappa_{j, t-1}\right)\left(\kappa_{\nu, t-1} \alpha_{1}^{-i}-\kappa_{\nu, t-i-1} \alpha_{1}^{i}\right) \\
=A^{3}\left(1-\alpha_{1}^{2 i}\right)^{2} \alpha_{1}^{2(t-1)-3 i+v} K^{*}(v)\left(K^{*}(j)-\gamma_{1} \alpha_{1}^{2}\right) \geq 0 .
\end{array}
$$

$\left.a_{2}\right)$ Let $i>j+1$. Then $I I I=\kappa_{\nu, t-i-1} I V+\kappa_{\nu, t-1} \kappa_{i, t-1} \alpha_{1}^{-j-1}\left(\kappa_{j, t-i-1}-\alpha_{1} \kappa_{j+1, t-i-1}\right)$ with

$$
\begin{aligned}
I V:= & \alpha_{1}^{-j-1} \kappa_{i, t-1} \kappa_{j, t-1}-\alpha_{1}^{-j} \kappa_{i, t-1} \kappa_{j+1, t-1} \\
& \quad+\alpha_{1}^{i} \kappa_{j+1, t-i-1} \kappa_{j, t-1}-\alpha_{1}^{i} \kappa_{j, t-i-1} \kappa_{j+1, t-1} \\
= & A \alpha_{1}^{-1} \kappa_{i, t-1}\left(K^{*}(j)-\alpha_{1}^{2} \gamma_{1}\right)+\alpha_{1}^{i}\left(\kappa_{j+1, t-i-1} \kappa_{j, t-1}-\kappa_{j, t-i-1} \kappa_{j+1, t-1}\right) .
\end{aligned}
$$

Using the presentation for the second parentheses from $a_{1}$ ) we obtain for $j \geq 1$ that $I V=A^{2} \gamma_{1}\left(1-\alpha_{1}^{2}\right) \alpha_{1}^{i-1}\left(\gamma_{1}-\alpha_{1}^{2(t-1-i)}\left(2-\alpha_{1}^{2 i}\right)\right)$. Inserting IV into III and assuming that $v \geq 1$ we get that

$$
I I I /\left(A^{3} \gamma_{1}\left(1-\alpha_{1}^{2}\right) \alpha_{1}^{i+v-1}\right)=C_{3}\left(\frac{C_{2}}{2 C_{3}}-\alpha_{1}^{2(t-i-v-1)}\right)^{2}+C_{1}-\frac{C_{2}^{2}}{4 C_{3}}
$$


with $C_{1}=2 \gamma_{1}^{2}, C_{2}=\gamma_{1}\left(1+\alpha_{1}^{2 i}+3 \alpha_{1}^{2 v}-\alpha_{1}^{2(i+v)}\right)$ and $C_{3}=2 \alpha_{1}^{2 v}$. Because $C_{2} \geq 2 C_{3}$ and $t-i-v-1 \geq 0$ a lower nonnegative bound of (4.12) is obtained if we put $t=i+v+1$. b) Let $i=j+1 \geq 2$ and $v \geq 1$. Using (4.10) it follows that $I I I=I I I_{1}+I I I_{2}$ with

$$
\begin{aligned}
I I I_{1}:= & \left(\alpha_{1}^{-i} \kappa_{i, t-1}+\alpha_{1}^{i} \kappa_{i, t-i-1}\right)\left(\kappa_{i-1, t-1} \kappa_{v, t-i-1}+\kappa_{i-1, t-i-1} \kappa_{\nu, t-1}\right) \\
& -\left(\alpha_{1}^{-(i-1)} \kappa_{i, t-1}+\alpha_{1}^{i} \kappa_{i-1, t-i-1}\right)\left(\kappa_{i, t-1} \kappa_{\nu, t-i-1}+\kappa_{i, t-i-1} \kappa_{v, t-1}\right), \\
I I I_{2}:= & -A\left(K^{*}(i)-K^{*}(0)\right)\left(\kappa_{i-1, t-1} \kappa_{\nu, t-i-1}+\kappa_{i-1, t-i-1} \kappa_{\nu, t-1}\right) \\
= & \alpha_{1}^{i+v-1}\left(-C_{4}+C_{5} \alpha_{1}^{2(t-i-v-1)}-C_{6} \alpha_{1}^{4(t-i-v-1)}\right)
\end{aligned}
$$

and $C_{4}=2 \gamma(1-\gamma)(\gamma+A)^{2}, C_{5}=\gamma(1-\gamma) A(\gamma+A)\left(1+\alpha_{1}^{2 i}\right)\left(1+\alpha_{1}^{2(\nu-i+1)}\right)$ and $C_{6}=2 A^{2} \alpha_{1}^{2(v+1)} \gamma(1-\gamma)$. Now $I I I_{1}$ is equal to the quantity III of case a) obtained by putting $i=j+1$ there. The calculations for case $a_{2}$ ) show that

$$
I I I_{1} / \alpha_{1}^{i+\nu-1}=\left(\gamma\left(1-\alpha_{1}^{2}\right)+\alpha_{1}^{2}\right)\left(C_{1}-C_{2} \alpha_{1}^{2(t-i-\nu-1)}+C_{3} \alpha_{1}^{4(t-i-\nu-1)}\right) .
$$

Consequently $I I I / \alpha_{1}^{i+v-1}=C_{7}-C_{8} \alpha_{1}^{2(t-i-v-1)}+C_{9} \alpha_{1}^{4(t-i-v-1)}$ with $C_{7}=2(\gamma+$ $A)^{2}\left(\gamma\left(\gamma-\alpha_{1}^{2}\right)+\alpha_{1}^{2}\right), C_{9}=2 A^{2} \alpha_{1}^{2 \nu}\left(\gamma+\alpha_{1}^{2}(1-\gamma)^{2}\right)$ and

$$
\begin{aligned}
C_{8}= & A(\gamma+A)\left(\left(\gamma\left(1-\alpha_{1}^{2}\right)+\alpha_{1}^{2}\right)\left(1+\alpha_{1}^{2 i}+3 \alpha_{1}^{2 v}-\alpha_{1}^{2(i+v)}\right)\right. \\
& \left.-\gamma(1-\gamma)\left(1+\alpha_{1}^{2 i}\right)\left(1+\alpha_{1}^{2(\nu-i+1)}\right)\right) .
\end{aligned}
$$

Note that neither $C_{7}$ nor $C_{9}$ depend on $i$. Then the maximum value of $C_{8}$ with respect to $i \geq 1$ is obtained for $i=1$. Because $C_{8}(i=1) \geq 2 C_{9}$ the lower bound of $I I I / \alpha_{1}^{i+\nu-1}$ is given by $C_{7}-C_{8}(i=1)+C_{9} \geq 0$.

\section{References}

[1] L.C. Alwan and H.V. Roberts. Time series modeling for statistical process control. J. Bus. Econom. Statist., 6:87-95, 1988.

[2] T.J. Harris and W.H. Ross. Statistical process control for correlated observations. Canadian J. of Chemical Engineering, 69:48-57, 1991.

[3] W. Jiang, K.-L. Tsui, and W.H. Woodall. A new SPC monitoring method: the ARMA chart. Technometrics, 42:399-410, 2002.

[4] T.Z. Lai. Control charts based on weighted sums. Ann. Statist., 2:134-147, 1974.

[5] M. Pawlak and W. Schmid. On the distributional properties of GARCH processes. J. Time Ser. Anal., 22:339-352, 2001.

[6] S.W. Roberts. Control chart test based on geometric moving averages. Technometrics, 1:239-250, 1959. 
[7] W. Schmid. On EWMA charts for time series. In: Frontiers of Statistical Quality Control, Lenz, H.-J. and Wilrich, P.-Th. (Eds.), Physica-Verlag, Heidelberg, Germany, 5:115-137, 1997.

[8] W. Schmid and Y. Okhrin. Tail behaviour of a general family of control charts. Technical Report 169, Europe-University, Frankfurt (Oder), Germany, 2001.

[9] W. Schmid and A. Schöne. Some properties of the EWMA control chart in the presence of data correlation. Ann. Statist., 25:1277-1283, 1997.

[10] W. Schöne, W. Schmid, and S. Knoth. On the run length of the EWMA scheme: a monotonicity result for normal variables. J. Statist. Plann. Inference, 79:289-297, 1999.

[11] Y.L. Tong. The Multivariate Normal Distribution. Springer, New York, 1990.

[12] D.G. Wardell, H. Moskowitz, and R.D. Plante. Control charts in the presence of data correlation. Management Science, 38:1084-1105, 1992.

Wolfgang Schmid

Department of Statistics

Europe University

PO Box 1786

15207 Frankfurt (Oder)

Germany

schmid@euv-frankfurt-o.de
Yarema Okhrin

Department of Statistics

Europe University

PO Box 1786

15207 Frankfurt (Oder)

Germany

okhrin@euv-frankfurt-o.de 